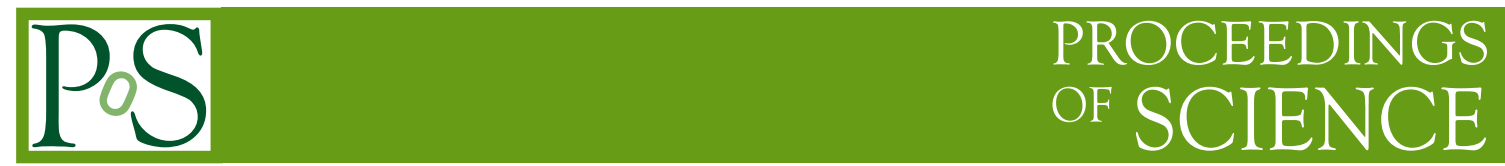

\title{
Adversity in life, sharing science
}

\section{Benoît Blossier*}

Laboratoire de Physique Théorique, CNRS, Univ. Paris-Sud, Université Paris-Saclay, F-91405

Orsay Cedex, France

E-mail: benoit.blossiereth.u-psud.fr

Building an inclusive society is an active field nowadays. Of course education is a key entry for people with disabilities to fulfill their citizenship. We present a couple of initiatives recently taken in France in that direction, by letting the pupils with disabilities discover and share science: in particular the operation "La Main à la Päte" promoted by Académie des Sciences and an effort undertaken by the Ministry of Education and scientists from CNRS to improve the instruction of teachers of physics and chemistry to work with those pupils.

The European Physical Society Conference on High Energy Physics 22-29 July 2015

Vienna, Austria

* Speaker. 


\section{Be at school with a disability}

I took the opportunity of a session "Education and Outreach" organised for the first time within the EPS-HEP cycle of conferences to let informed my scientific community about what doing science despite a disability means in practice, even if the context is a priori not encouraging, especially at school. Obviously, I am not familiar with science of education, even less with the question of pedagogical accessibility, I can only advocate my daily life as a visually impaired person. Indeed, I give lectures to students finishing their master or doing their $\mathrm{PhD}$, they are so highly interested by what is taught that presenting something pedagogical to them is not really difficult. However I sincerely hope that my assertions will shed light on issues and progresses towards an inclusive society. My research activities are devoted to particle physics, more precisely flavour physics and lattice QCD: during the conference I showed a poster on results recently obtained in $B$ physics [1]. I defended my $\mathrm{PhD}$ thesis in 2006 at LPT Orsay after graduate studies at Ecole Normale Supérieure in Paris. Then I spent 2 years as a post-doctoral fellow at DESY Zeuthen before getting a permanent position at CNRS. I am the youngest student of my ENS physics class to have been appointed: it is somehow a pride. From the medical point of view I have a severe to deep visual impairment that is caused by the Peters Syndrome: consequences are an opaqueness of the cornea, a nystagmus (uncontrolled motions of the eyes), a high pressure in the eyes leading to a glaucoma and detachment of the retina, possibly irreversible. It goes also with a very strong myopia and a tubular vision. One year ago I noticed the appearance of a scotoma (a hole in the field of vision), in the central region of my remaining eye, indicating that I was on the way to loose my fight against pressure: it was urgent to take more efficient eye lotions. I do not recognise faces further than 1.5 $\mathrm{m}$ and I cannot read what is written on badges without putting my eyes extremely close to them. A very important point is that working on usual supports (paper, computer screen) is still possible, my far vision was quite comfortable until 2014 thanks to the use of a monocular: it is helpful to read slides shown by colleagues in conferences or to find the right gate in airport terminals. Nowadays my locomotion in an unknown environment is more and more hesitant, I am close to use a blind cane under certain circumstances, in particular to protect myself against crushes in a big crowd.

\subsection{Schooling achievement of a young disabled pupil}

In my opinion social pressures are at work for the most beneficial education as possible to a pupil with a disability, at pretty early age already. I am one of those "heirs" discussed by Pierre Bourdieu [2], too: my both parents were teachers in the country side, I learnt reading with my mother: hence I could not fail that crucial learning phase. Reading book characters were large enough for me to distinguish them without any trouble, I was writing characters between the lines of my copy book, I was keen to perform arithmetic operations and the life in a small country school favored my self-fulfillment. From the beginning my family was seeking to satisfy my curiosity in my (more sound than visual) environment. The identification of bird songs, using audio recordings that were perfectly adapted to kids like me because I was told small stories for each species presented, has enhanced a lot my attention to listening: I consequently worked very early that means of compensation in a highly efficient way because I was doing that effort as a volunteer, so to speak, as a distraction. At the same time I was reading with a lot of passion ornithology essays: I was getting unconsciously used to characters of printing size. I discovered Europe and its geography 
thanks to distributions maps of the avifauna within Western Palaearctic, then I was able to follow the geopolitical news, particularly rich at the end of the 80s. It is appropriate to assure an early immersion of the child in an ordinary (unprotected) environment. In my case I went to an ordinary establishment in the main city of my department, to which a specialised structure was associated: I was sharing my time between the ordinary class and that structure where I learnt Braille and typing: I could convince myself that I could integrate in the social world of valid people while I was getting the living wage to compensate my disability in assimilation and restitution of knowledge. It is necessary to try equipments helping the schooling and be careful to bring very fast changes in case of failure. For instance I used at some point a monocular: for the first I was able to read on the blackboard. However, this deep satisfactory moment was very short when I discovered that writing up a surface freshly washed with a sponge, using a chalk, was finally unreadable. I abandoned an intensive use of my monocular to privilege taking notes by listening what the teacher was saying. Teachers have to individualise their teaching if it is requested: sometimes the adaptation is simple. When I learnt how to divide two numbers, place on the paper elements of a division (dividend, divisor, quotient, rest), with only a sound source of information, was not trivial the first time: my teacher came to my desk and explained me again what I had not seen, to my great profit.

\subsection{Schooling achievement of a teenager in high school}

A key point is use in a smart way compensation means of different kinds. Following a request of my French teacher, I was typing my dictations although I was shameful to do that because the noise of the typewriter was certainly bothering my classmates attention. Nowadays, in our world of computers, I could fully accept it. As I arrived at school a light was shining on my desk and, at home, I used a tilted desk. When I was 13 we installed that system in each classroom: a wooden desk was set on my table. A first stop fixed on the bottom of the plane surface was retaining books and copies and a second one, fixed under the desk, avoided to drag it on the table once the stop arranged against the edge of the table. Thus my back was not too crooked when I bent to put my eyes a few centimeters of the work plane. At a first stage it is welcome to individualize punctually the teaching but I would tend to diminish it if it is too stigmatising. When I started to learn English, I implicitly wished spelling of the first English words that we had to write on our notebook, like "first name": that's why nothing occurred, probably. Two years later I decided to express myself during a math class, because our teacher persisted in refusing to orally describe what she was writing on the blackboard. Finally we decided that she would come to my desk and explain again, with a big pen, what she had to demonstrate a few minutes before to the whole class on the blackboard: I would have preferred to take notes by myself. When a teenager is more mature, it is time to encourage him/her to take initiatives with a "demanding caring" assured by the teaching team. Indeed, at the end of the day, that rule of life applies in the real world. I copied from my neighbor's sheet experience schemes that were drawn on the blackboard without the teacher of physical and chemistry bothered to comment them, or at least it was too elliptical: therefore he was not aware of my difficulty but it was easy to circumvent. Note that this support among students is easier to organise in high school because they are more mature than in collège and are able to make fairly quickly sketches or tables, so that the student who is waiting for the piece of information is rarely out of step with the rest of the class. Sessions of lab work, though they were quite challenging for me, took place smoothly because it was clear how to share the tasks with my 
binomial partner: I was letting him most of the handlings and I was writing the report comments after an interpretation of the results and observations in collaboration. It is crucial to pay a special attention to the choice made by the pupil in future studies: discuss pathways and opportunities without hiding additional difficulties to overcome due to the disability. A successful day is not necessarily that of the next day, there is no free lunch for an orientation failure: one year counts twice in terms of efforts and accumulated fatigue, if not sometimes more.

\subsection{Valuable course of a disabled student at the University}

Technology developments allow an easy access to pedagogical resources: filmed lectures, typed notes given well in advance, digitised books (voice synthesis, e-learning). As far as the student is concerned, I strongly recommend to prepare in advance tutorials, in order to get used to make any write-up, whatever the medium. Indeed, it happens often that students with disabilities write their exam or competitive examination copies with a secretary. This is not trivial because it means that the expression of their thoughts is transmitted by a third party: there is a kind of delay effect and, as we know, time is a relevant factor during evaluation sessions. The applicant will save time if he/she has already practiced a collaboration with a secretary.

\section{Make science accessible to outsiders}

\subsection{Train teachers}

In France, the so-called "law of $11^{\text {th }}$ February 2005", setting the framework to fulfill the citizenship of disabled people, must have as a corollary some initiatives improving for disabled pupils the benefit of their schooling. The first answers are employing human helpers and funding expensive devices. Unfortunately, they are not enough: one forgets very often to let aware of the obstacles faced by any disabled pupil to pass the requirements of subjects taught. Ministry and parliament reports point that teachers are not sufficiently informed and trained about adapting the pedagogy of their lessons [3], [4], [5]. Experts advice to reinforce the autonomy of the pupil and highlight his/her intellectual work: those two skills are particularly sought by employers. I proposed to prepare a booklet offering a couple of indications to teachers who are supposed to welcome in their classroom sensory impaired pupils or with specific troubles in learning. Among other subjects, Mathematics, Physics and Chemistry, Biology and Geology are discussed in the resources. Different disabilities can be overcome by various pedagogical adaptations: a dyslexic pupil will prefer to work from schemes than from texts that have a high cognitive cost, it is better to use an inductive approach with deaf pupils due to their lexical poverty. In function of the subject, different questions can be asked about methods of teaching to disabled pupils:

- in Mathematics, how to learn the space geometry to a visually impaired kid?

- in Experimental Science, how to put disabled pupils in a good position to be active in a small group in the framework of an investigation process?

Two or three "sequences" per subject are selected thanks to the quality of their building and the richness of their pedagogical content. The seven years of "collège" and "lycée" are covered. The sequences were really tested in classrooms, as well as their adaptations. In Figure 1 we show two examples of adaptations that are not difficult to propose: in an introductory lesson of chemistry, 
clearly indicate the safety recommendations, while dyslexic children memorise well the informations presented on a heuristic card. After publication of that work by the French ministry of education [6], I was informed about proposals of activities to train teachers in a very practical way. The most emblematic one was made to prepare applicants from Université Paris-Sud to answer questions concerning inclusive society during their oral session of the Biology teachers competitive exam: they had to study a series of documents, including a sequence of the booklet. The target is now to improve the training of teachers on that problematic by identifying experts in pedagogical accessibility of physical science to build a national network and training trainers. I am convinced that the mutualisation of good practices should be a long-term policy of any educative system.

\subsection{Let disabled kids do science in the framework "La Main à la Pâte"}

Asking the question whether teaching science with the investigation approach can help pupils who face difficulties, Georges Charpak, Pierre Léna and Yves Quéré answered that ii is beneficial because it is based on the existence of a "tiers objet", for instance the shadow of a person on the floor or the puff of wind, that catalyze the questioning and generate a powerful mean of satisfying the curiosity of the kid [7]. They were told that pupils with a motion or a mental disability were profiting of those lessons as well. In 2010, the decision was taken to propose the framework "La Main à la Pâte" to establishments where a high number of disabled kids were in school. The protocole is well-defined from the science of education point of view and teachers work together with scientists. Pupils discovered science with sequences like "the Sun and us", "float or sink" or "the electric manege" shown in Figure 2. Conclusions of the experience are the following [8]:

- It is possible to organize the work in small groups, something known as particularly positive: pupils collaborate among themselves, they share the tasks according to their abilities.
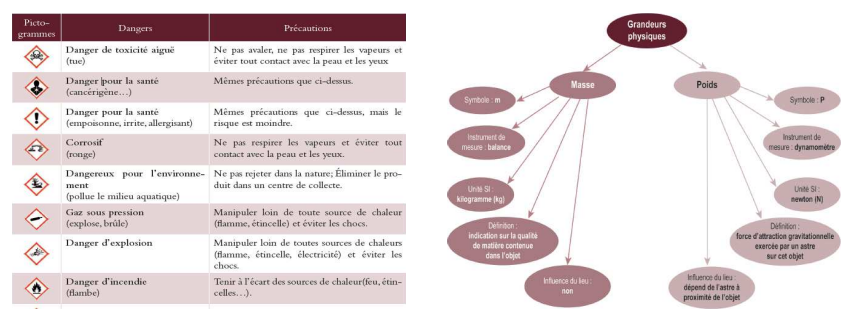

Figure 1: Safety recommendations given at the first chemistry lesson (left panel); heuristic card to memorise characteristics of weight and mass (right panel).
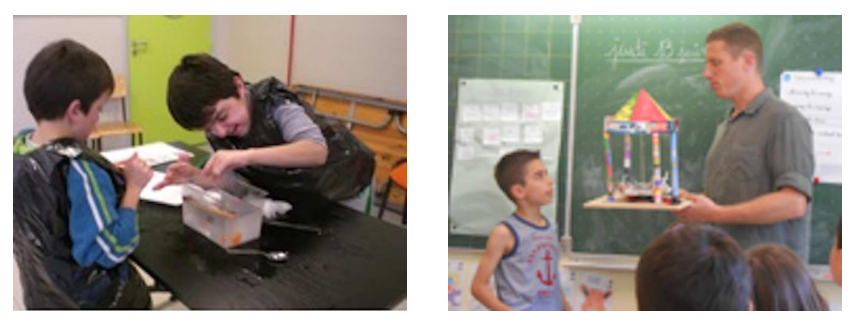

Figure 2: Sequences proposed by "La Main à la Pâte" to pupils with a disability: "float or sink" (left) and "the electric manege" (right). 
- Handling is an excellent factor to make pupils involved in the work, even if their disability was sometimes a real obstacle to properly handle the elements of an experiment.

- The learning is long-lasting: one year or even two years after the work, pupils remember for instance the condition to let a light shine (closed electric circuit) or the role of the air to explain why an object is floating.

- They accept the "try/error" process: they understand that an error in explaining a phenomenon, or to predict it, is not a failure, it is associated to the scientific approach. Because of their usual bad self-respect, it is a non negligible benefit.

- Conceptualization is as difficult for disabled pupils as for everybody. A convincing example is the position of the shadow with respect to the person, as shown in Figure 3.

- There was the need to adapt the work. Time factor is not to neglect: sequences take typically twice longer than for pupils without disability. Specific equipments are sometimes required and rituals favorise the learning: pupils remember what they did last time, what they will do this time, to answer to which question and so on. Some of those adaptations are efficient to other pupils.

After a couple of years spent to investigate that question, I came, too, to the conclusion that thinking about the pedagogical accessibility proceeds like a zoom effect on learning troubles faced by almost every pupils. I will repeat and illustrate that message again and again within my community.

\section{References}

[1] B. Blossier, "Strong decay of scalar B meson", PoS (EPS-HEP2015), 598 (2015).

[2] P. Bourdieu and J.-C. Passeron, "Les Héritiers, Les Etudiants et la culture", Paris, Minuit, Coll. "Le sens commun" (1964).

[3] C. Becchetti-Bizot et al, "La scolarisation des élèves handicapés, formation et accompagnent des équipes pédagogiques du second degré", Rapport au Ministre de l'Education Nationale, 2008.

[4] A. Rebeyrol et al, "Discriminations à l'Ecole", Rapport au Ministre de l'Education Nationale, 2010.

[5] P. Blanc, "La scolarisation des enfants handicapés", Rapport au Président de la République, 2011.

[6] N. Audoin et al, https://eduscol.education.fr/ash-ressources-disciplinaires (2013).

[7] G. Charpak, P. Léna and Y. Quéré, "L’enfant et la science", Ed. Odile Jacob (2005).

[8] M.-H. Ferrand-Heitz and E. Saltiel, BUP 976, 971 (2015).

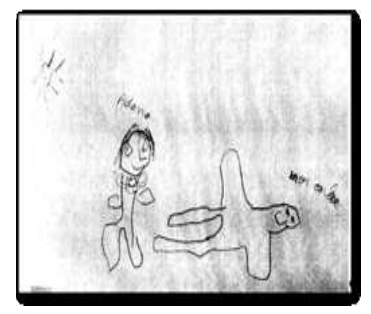

(a)

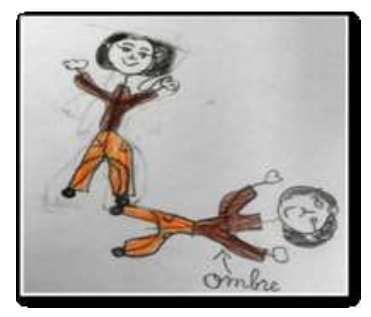

(b)

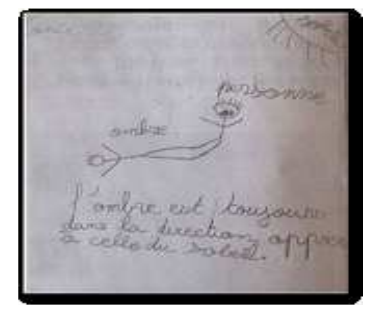

(c)

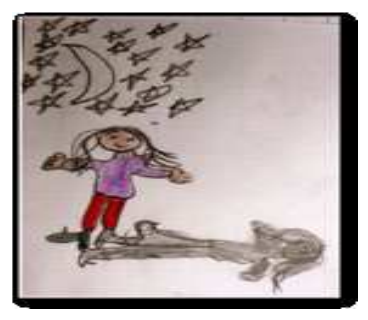

(d)

Figure 3: Schemes drawn by pupils of the relative position of a person with his/her shadow; (a) and (b) at the beginning of the lesson "the Sun and us", (c) and (d) at the end. (a) and (c) are drawn by a disabled pupil, (b) and (d) are drawn by a lambda pupil. 